\section{Functional Neurorehabilitation - The Locomotor Quadrupedal Animal Training Adapted to the Bipedal Human}

Ângela Martins

DVM, CCRP, ESAVS Member of AARV AND IARVT Department of Veterinary Sciences University of Lusófona de Humanidades e Tecnologia Campo Grande 376. 1749 - 024 Lisboa Portugal.

Contact information:

Ângela Martins

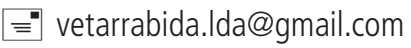
Keywords
Functional Neurorehabilitation; Locomotor trainning; Neural plasticity; neuromodolation; Supraspinal Input; Spinal cord and treadmill.

\section{Introduction}

A rigorous rehabilitation program, results in a marked recovery with locomotor training (LT) (Figure 1), associated with electrical stimulation (transcutaneous, intraspinal or epidural) and the administration of pharmacological agents in the motor function in the quadrupedal animal as in the bipedal human (Olby, 2014). These rigorous programs can be implemented in complete spinal cord lesions, since the spinal cord has a degree of automaticity, these meaning that the spinal cord has the ability to interpret complex sensory information for posture and locomotion (Angeli, Edgerton, Gerasim- 


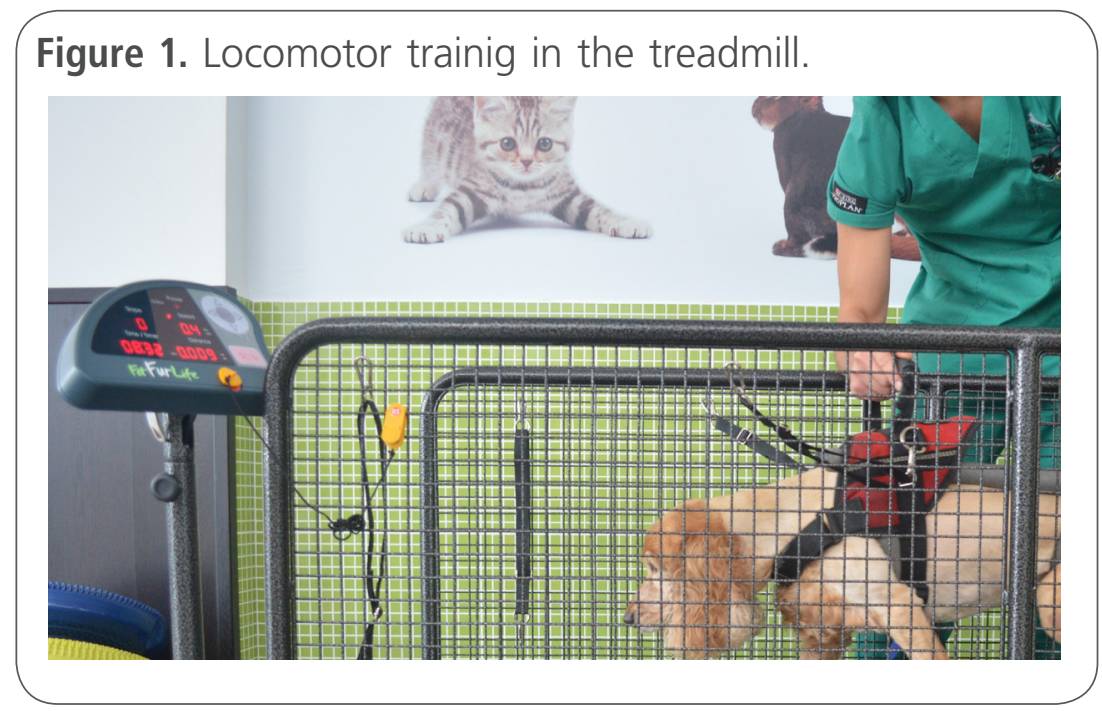

enko, \& Harkema, 2014; Barros \& Pinheiro, 2014; Garcia, Alias, Truorg, Shah, Roy, \& Edgerton, 2015; Chang, 2014; Davies, 2015; Ditunno, Cardenas, Dalal, et al, Advances in rehabilitation management of acute spinal cord injury, 2012; Gerasimenko et al, 2010; Lavron et al, 2015; Lavron, Gerasimerko, et al, 2014; Musienko, Courtine, et al, 2012 ; Nielsen et al, 2015; Solopova et al, 2015; Tansey, 2010; Thompson \& Walpaw, 2014; Zhong, Roy, Leon, \& Edgerton, 2012).

Given these data, the LT has a key role, requiring:

- Automaticity in the activity of the spinal cord;

- Automatic sensory input from the spinal cord;

- Neuromodulation;

- Ability to learn through the locomotor circuitry of the spinal cord

- Locomotion control through the descendants pathways of upper motor neuron (UMN). The spinal cord automaticity is a property of self-regulation and functionality, without conscious control (Roy, Harkema, \& Edgerton, 2012). Studies in human medicine, demonstrate the property of automaticity, neuromodulation of the afferent inputs for controlling the coordination of circuits among limbs, coordination of flexor and extensor muscles of a limb, and control of the speed, direction, and balance of the gait. The central pattern generators (CPGs), can coordinate the motor input based on the received sensory inputs and still premeditate the sequence of logical action (Davies, 2015; Roy, Harkema, \& Edgerton, 2012). The CPGs are an important component of the neural circuitry, located within the lumbosacral region of the spinal cord to promote the control of gait and posture (Roy, Harkema, \& Edgerton, 2012). The fictive locomotion is a combination of a sensory input process and the CPGs activity themselves. Human spinal cord is proven to be autonomous (Davies, 2015; Roy, Harkema, \& Edgerton, 2012).

\section{Locomotor Training}

The $L T$ is a rehabilitation strategy designed to regain postural control, balance, postural standing, gait, independence of function and quality of life after neurological injury. The LT is an intervention that results in the activation of the neuromuscular function to recover motor function, in order to get our target, that is to retrain the nervous system to recover a specific function (Harkema \& Behrman, 2012; Harkema, Schmidt, et al, 2012; Harkema, Schmidt-Read, Lorenz, \& Edgerton, 2012). The activation function of the neuromuscular system, occurs during the repetition and progression of the LT, in order to obtain neural plasticity. This theory is held in the hu- 
Vol. 8 No. 179 doi: $10.3823 / 1778$ man biped by evidence (Dragone, 2015; Harkema \& Behrman, 2012; Harkema, Schmidt, \& al, 2012). The objectives of the LT are:

- Increase the ability to bear the weight with the limbs;

- Increase the afferent pathways to increase the reflex arc;

- Optimize the posture;

- Maximize the recovery and minimize the compensation (Harkema \& Behrman, 2012).

\section{Brain-Machine Spinal Cord Interface (BMSCl)}

Edgerton and Panag Gad, performed a machine named $\mathrm{BMSCl}$, which uses electromyography signals from the forelimbs to regulate and promote spinal cord stimulation, in order to promote the movement of the hindlimbs. The electromyography of the forelimbs is important because these persistent inward current (PICs) enter in the normal course cycle of the quadruped locomotion (Gad, Edgerton, 2012). The spinal cord neuromodulation, allows the control of movements in humans with other neuromotor disorders such as cerebral vascular stroke, and Parkinson's disease.

\section{Fictive Locomotion}

Grillner and Zangger demonstrated that a tonic stimulation, stimulates the reflex arc, starting with the stimulation of the dorsal horn of the spinal cord to induce the fictive locomotion (Figure 2), or locomotion without supraspinal input (Musienko, Courtine, et al, 2012; Roy, Harkema, \& Edgerton, 2012).

It was assumed that this movement is a reflex and it is not functional, although it can bring support, postural standing and gait (Roy, Harkema, \& Edgerton, 2012). In this locomotion the reticulospinal neurons (RSN) and the mesencephalon locomotor region (MLR), have a key role. We can also associate a residual or regenerative pathway that allows propagation of PICs and may amplify the spinal cord automaticity and thus the control of locomotion, posture and learning capacity (Knikou, 2012). In quadrupeds, the motor cortex plays a minor role in the control of locomotion (Roy, Harkema, \& Edgerton, 2012).

\section{Descendants UMN Tracts}

The MLR and RSN have an extreme importance on movement, as we also know that the MLR is controlled by inhibition, and therefore the initia-

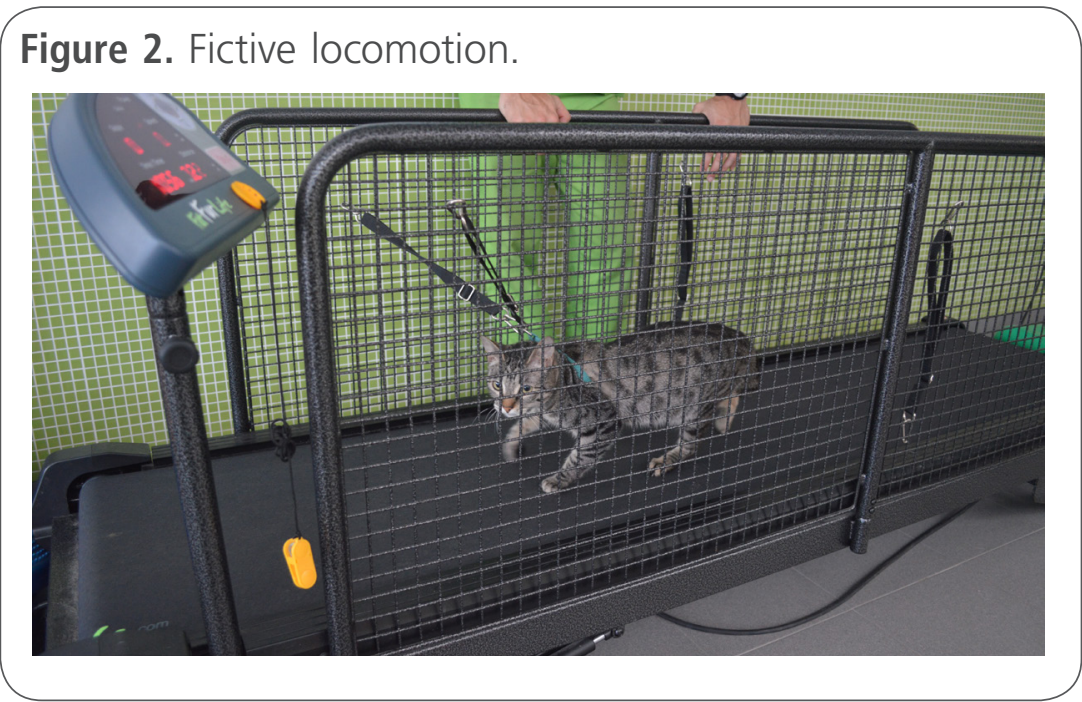


tion of locomotion can be induced by the desinhibition of RSN, vestibulospinal neurons (VSN) and rubroespinhal neurons (RuSN), which are activated in a rhythmic form during locomotion.

The VSN are activated by the postural standing, while RuSN and reticulospinal neurons (RSN) are activated at maximum of the protraction phase in the locomotion cycle. Although the VSN facilitate the extensor motoneurons, and RSN facilitate flexion with inhibition of extension. The corticospinal neurons (CSN) and RuSN facilitate flexor motoneurons and are the descending pathways of UMN that have neuromodulative function during specific stages of locomotion.

The rhythm and strength of the descending UMN tract is influenced by the ascendant input of the spinal cord circuitry (Roy, Harkema, \& Edgerton, 2012).

\section{Somatosensory Input}

The combination of CPGs and MLR are the basis of neural control strategies and automaticity of the spinal cord. Every human neural circuitry has this base, has indicated earlier, are central circuits of rehabilitation intervention and LT (Dietz, 2012; Roy, Harkema, \& Edgerton, 2012; Harkema, Schmidt, et al, 2012; Knikou, 2012).

In the human biped, afferent inputs are essential for CPGs and for the effectiveness of LT (Dietz, 2012). In the human biped, without the locomotor activity induced by functional training, the muscles go into exhaustion in assisted locomotion. By this, we can conclude that a workout with appropriate proprioceptive input and beginning in an early stage after a spinal cord injury, is the only way to prevent neural dysfunction (Dietz, 2012).

The neural mechanism associated with posture and locomotion is a rehabilitation method that is essentially to the LT, as well as the electromyostimulation, which allows a direct stimulation of afferent fibers at the level of the dorsal horn of the spinal cord (Musienko, Courtine, et al, 2012).

The postural system depends on a world of inte- grations involving vision, the vestibular and somatosensory inputs (Musienko, Courtine, et al, 2012).

The orientation of the head is established with the vestibular and visual information while the somatosensory information allows the stabilization of the body during the postural standing (Musienko, Courtine, et al, 2012).

\section{Bipedal Training}

An example of a LT protocol is indicated by Roland R. Roy and Huizong, which consists in training the animals to a bipedal training after 7 days post-surgery, for 30 minutes per day, 5 days a week, for 6 months. Most of the patients, which are cats, demonstrated fictive locomotion after 3 months (Zhong, Roy, Leon, \& Edgerton, 2012).

This type of training is clearly applicable in human biped and even children with or without incomplete or complete spinal cord injury (Zhong, Roy, Leon, \& Edgerton, 2012).

\section{Specific Exercises of Locomotor Training}

The LT consists in a fortification exercise, which consists in a short time stimulation of the patient's maximum performance, such as:

- Sit to rise;

- Rise to sit;

- Sit and stretch the trunk;

- Sit to rise, and initiate gait;

- Place the center of gravity to the left, to the right and diagonally. All these exercises must have assistance, but independence of some areas of the body should be stimulated, therefore to an independent control (Bostrom et al, 2014; Harkema, Schmidt, et al, 2012). After this type of kinesiotheraphy exercises, exercises in the treadmill should be performed. The speed of the treadmill varies according to the activation capacity of the neuromuscular system (Harkema, Schmidt, et al, 2012). Every day, the technician assesses the patient and develops a plan 
with therapeutic goals, implementing an individualized protocol (Harkema, Schmidt, et al, 2012). The therapeutic goals are:

- Balance (Figure 3);

- Center of gravity transference;

- Carrying out daily activities;

- Ambulation (Harkema, Schmidt, etal, 2012). In the case of human biped, the use of orthoses should be reduced for the lower limbs. The LT must be conducted intensively, 5 times a week and with 90 minute sessions (Harkema, Schmidt, et al, 2012).

\section{Memorization of the Locomotor Training}

The LT implies retraining the nervous system, in a controlled environment, to achieve a postural standing and involves verbal and manual stimulation by the technician. The memorization of the LT involves:

- The retraining of postural standing;

- The adaptation to postural standing;

- The retraining of the gait;

- Gait sessions of at least 55 to 60 minutes;

- Retraining to sit and gait after sitting. Retraining gait sessions should have a gradual duration of 20 a 60 minutes, in order to stimulate the neuromuscular response and sensorymotor experience (Harkema, Schmidt, et al, 2012; Steinberg, 2013). The training must take into account the motor control of the trunk and the limbs should support $60 \%$ of body weight. In more advanced LT, is intended to stimulate the gait at a terrestrial level, followed by a 60 to 90 minutes period of performing daily tasks (Harkema, Schmidt, et al, 2012). During a LT session of 90 minutes, the technician aid should be gradually dispensed and the use of distal member orthoses should only be used at home. The patients can not finish the training until they improve their performance and are monitored every 20 minutes. The monitoring consists in the neurological examination of motor function, balance, autonomic function and gait parameters. Usually training has a duration of 6 to 12 months and two technicians are needed by patient (Harkema, Schmidt, et al, 2012).

\section{Locomotor Training and Neurorehabilitation}

The LT consisting on the support of the body weight on the treadmill, is a used method in the

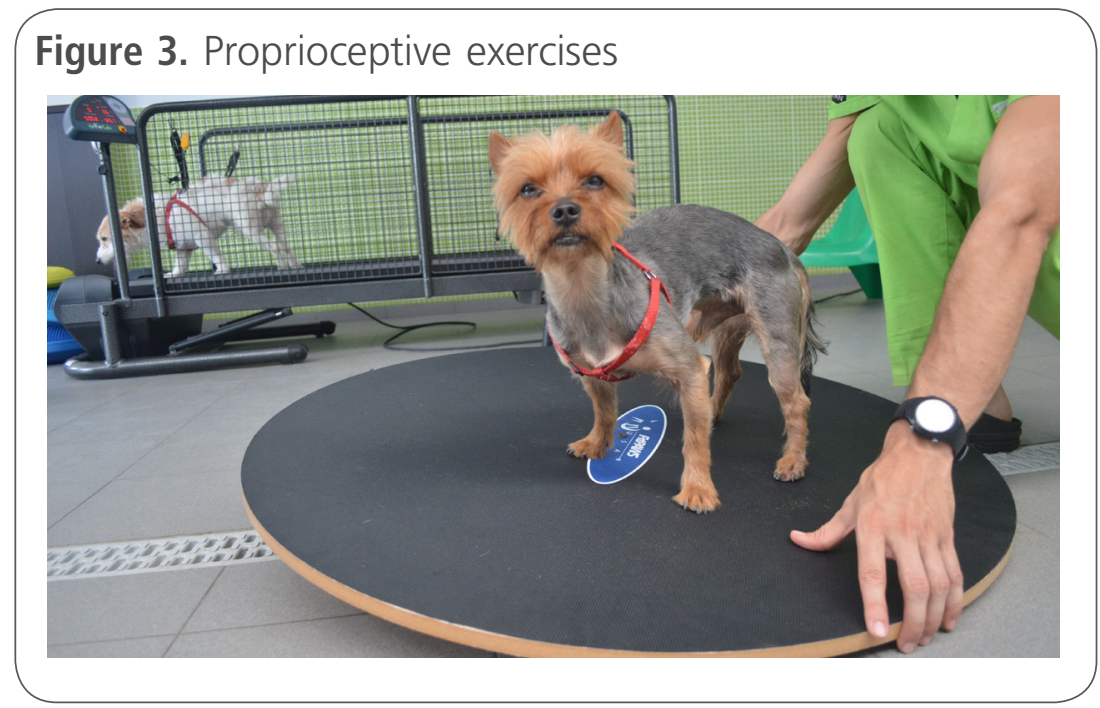


therapeutical plan for the recovery of spinal cord injury, cerebrovascular disease and Parkinson's disease and has been shown to be extremely effective (Alexeeva, et al., 2011; Duffell, Brown, \& Mirbagheri, 2014; Duffell \& Mirbagheri, 2015; Field-Fote \& Roach, 2011; Takao, Tanaka, Yanagi, et al, 2015; Werning, Muller, Nanassy, \& Cagol, 1995; Wirz, Zemon, Rupp, Scheel, \& Hornby, 2005; Visintin \& Barbeau, 1989). This method is called "Functional Organization of the Brain" and consists of a specific test used in neurorehabilitation techniques (Duffell \& Mirbagheri, 2015; Takao, Tanaka, Yanagi, et al, 2015). A study performed by Yanagi, indicates that 13 LT sessions per month, with a rate of 3 sessions per week, lasting 20 to 40 minutes per session, results in a clear recovery and in a accelerated gait composed by large strides, indicating an improved gait (Takao, Tanaka, Yanagi, et al, 2015).

This type of LT reduces the abnormal neuromuscular activity and although it is not directly related to functional improvements, allows improving of muscle strengthening and promotes balance (Duffell \& Mirbagheri, 2015).

\section{Spasticity, Pain and Proprioception}

In the human biped $70 \%$ of patients with spinal cord injury develop spasticity 2 to 6 months after the occurrence of spinal cord injury, associated with increased tendon reflex and muscle tone, and muscle spasm (Hagen, 2015; Roy \& Edgerton, 2012).

Most studies report to PICs as an important component in the occurrence of spasticity (Roy \& Edgerton, 2012). A increase of chlorine in motor neurons, after a spinal cord injury, promotes a change in sodium and calcium channels, this alteration in turn, contributes to the hyperexcitability of spinal reflex pathways. As a result of these cellular neurophysiological alterations, changes occur in the PICs and consequently neural depolarization time increases, leading to increased spasticity and reducing the reciprocal inhibition (Roy \& Edgerton, 2012).
The type of spasticity present in the human is equal to the cat (Roy \& Edgerton, 2012).

Gabapentin and baclofen are two of the most frequently used active principles in the pharmacological treatment of spasticity, as well as the toxin produced by clostridium botulinum, which is not recommended for prolonged treatment (Roy \& Edgerton, 2012).

\section{Robotic Training (RT); Endurance Training; Transcutaneous Electrostimulation}

When the patient is able to support its own body weight, the endurance training must be started, consisting in the stimulation of gait for long periods of time without reaching the patient's maximum capacity.

Robotic training is a new approach in neurorehabilitation that should be taken into account. Robotic systems as the Lokomat aim to promote and increase the motor function (Knikou, 2012; Nas, Yazmalar, Aydin, Ones, et al, 2015).

Robotic training consisting of 60 sessions of 1 hour per day, for 5 days a week, had shown an increase of $30 \%$ in the speed of gait and the capacity of the body weight bearing in the patients that underwent this training (Knikou, 2012).

Throughout the LT, transcutaneous electrical stimulation should be performed, by being considered neuromodulatory of the posture and neural circuitry involved in locomotion. This modality is of great importance in human training for aiding in the reduction of spasticity (Hahm, Yoon, \& Kim, High frequency transcutaneous eletrical nerve stimulation alleviates spasticity after spinal contusion by inhibiting activated microglia in rats, 2014).

For the LT to be successful, it must be started as soon as possible and with high intensity exercises (Ditunno, Cardenas, Dalal, et al, Advances in rehabilitation management of acute spinal cord injury, 2012; Hettlich \& Levine, 2012; Kim B., Ahn, Yoon, et al, 2014; Oosterhuis, Ostelo, et al, 2013). 


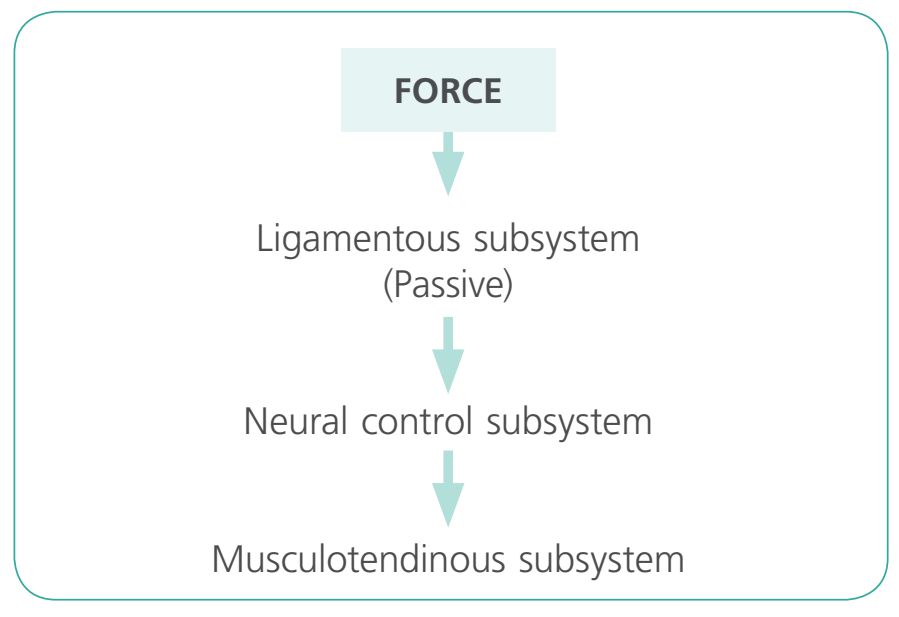

In $80 \%$ of human biped patients present pain after spinal cord injury (Hagen, 2015), since they have pain with or without neuropathic pain (Hagen, 2015; Hodges, 2014) thereby to reduce the chronic pain is important to decrease the primary neurological damage and undertake prevention for secondary injury due to hypoperfusion, ischemia and apoptosis (Hagen, 2015).

During the FNR protocol, we must carry out passive kinesotheraphy exercises to resolve contractures, muscle atrophy and pain, this is the first approach of the FNR training.

The protocol will be when in spinal shock, or flaccid muscle once a day, while in situations with muscle spasticity, two to three times daily(Nas, Yazmalar, Aydin, Ones, et al, 2015), followed by passive stretching, in those two cases.

We should start as soon as possible fortification exercises associated with eletromyostimulation. Then associate the proprioceptive training stimulating the patient to sit in a progressive manner, so he doesn't present a hypotensive crisis. When the patient already carries out the exercise to sit correctly, we will start the balance proprioceptive exercises(Nas, Yazmalar, Aydin, Ones, et al, 2015).

During LT, progressively, the slope of the treadmill should be increased, which will lead to greater resistance to movement and consequently increase of motorneural activity and the activation of cho- linergic pathways (Kirkby K., 2014; Tillakaratne, Edgerton, Roy, et al, 2014).

The LT should respect the Panjabi's model or spinal stability model. The Panjabi's model consists in the association of 3 subsystems. The passive or ligamentous subsystem, the active or musculotendinous subsystem and the neural control subsystem (Vangelder, Vaughn, \& Hoogenboom, 2013).

Other objectives of rehabilitation are to promote the range of motion (ROM) and reduce pain (Millis, Garr, \& Weng, 2013). Exercises such as climbing stairs and 90 minutes of marching on different types of surface, help to increase ROM (Kirkby K. , 2013; Kirkby K. , 2014), in order to work all muscle groups (Stanley \& Kim, 2013).

\section{Lasertherapy and Locomotor Training in Quadruped Animals}

The LT in quadruped animal, after section of the spinal cord, must take into account the forelimbs, as these influence the recovery of the hind limbs, being the synchronized training of the forelimbs with the hindlimbs more effective than bipedal training (exclusively of the hind limbs) (Shah P., Gerasimenko, Roy, \& Tillakaratne, 2013).

This type of training leads to increased excitability of motor neurons, especially the interneurons that connect with the proprioceptive neurons (Shah P., Gerasimenko, Roy, \& Tillakaratne, 2013). So the afferent feedback of the forelimbs influences the activity of the hind limbs, this being achieved by direct activation of proprioceptive descending pathways, interactions through inter-conecting short propriospinal axon pathways and also by ascending supraspinal pathways that culminate in the locomotor region of the brainstem or cortex, place from which the bulbospinal pathways descend. This phenomenon is called the spino-bulbo- spino loop, which is activated by different inputs from the forelimb, which in turn are projected to the spinal cord and will stimulate lumbosacral motor neurons, explaining the importance of the quadrupedal training 
(Shah P. , Gerasimenko, Roy, \& Tillakaratne, 2013).

The class IV lasertherapy is an essential modality in rehabilitation, that in veterinary medicine has already been proved its benefits. It allows a reduction in the inflammatory cascade and promotes the granulation tissue formation and epithelialization and may be applied to human medicine since the incidence of postsurgical infections is 1 to $2.4 \%$ (Cross, 2014; Medina, 2013; Newark, 2012; Kirkby K. , 2014; Rossmeisl, White, et al, 2013).

\section{Discussion}

In this article we intend to explore the constant relationship between neuroanatomy and neurophysiology of dog and cat to the human, showing that both are similar, and therefore for a FNR, locomotor training is an essential approach. The locomotor training can stimulate afferent inputs (Dietz, 2012) that stimulate the motor inputs and their coordination, that arises from the central pattern generator (CPG) (Davies, 2015). Therefore that is an important component of the CPG located at lumbosacral level (Roy, Harkema, \& Edgerton, 2012), thus allowing the importance of the bipedal locomotion training (Zhong, Roy, Leon, \& Edgerton, 2012). In this article it is proved that the realization of locomotor training after a spinal cord injury, stroke or Parkinson's disease should be implemented as soon as possible, within the level of each disease, and intensely with several repetitions, as the goal is to re-train the nervous system (Ditunno, Cardenas, Dalal, et al, 2012; Harkema \& Behrman, 2012; Harkema, Schmidt, \& al, 2012; Hettlich \& Levine, 2012; Kim B. J., Ahn, Yoon, et al, 2014; Oosterhuis, Ostelo, et al, 2013; SJ, M, DJ, Edgerton, et al, 2012;) promoting neuronal plasticity. The bipedal training is fundamental for the fictive locomotion (Harkema, \& Edgerton, 2012; Musienko, Courtine, \& al, 2012; Roy,). This article also proves that after the onset of voluntary or involuntary movement, being this conscious or not, we have to stimulate the RSN and the MLR, to allow a further spread of the PICs, and therefore the automaticity of CPGs (Knikou, 2012). This is one of the basis of neural control and strategies, and its allowed by quadrupedal locomotor training (Dietz, 2012; Harkema, Schmidt, \& al, 2012; Knikou, 2012; Roy, Harkema, \& Edgerton, 2012) The rhythm and intensity strength of locomotor training, allows a greater stimulation of the sensory afferent inputs of pelvic members, which increases the reflex arc and the CPGs of lumbosacral region. Then in the quadrupedal training we stimulate the connective interneuron of the forelimb and hindlimb, allowing a greater coordination and neuromodulation, both in quadruped animal as in the human biped. Both in quadruped as in human biped, the loop stimulated by the sensory inputs of the forelimb (spinal cord to cortex (corticospinal nuclei), and to spinal cord in the human), or in the spinal cord loop to brainstem (rubrospinal nuclei in the dog, and in the cat rubrospinal and corticospinal nuclei) to spinal cord, it will stimulate the UMN descendants pathways, stimulating lumbosacral neurons, which are stimulated by afferent inputs of pelvic member, improving mobility (Dietz, 2012; Harkema, Schmidt, \& al, 2012; Knikou, 2012; Roy, Harkema, \& Edgerton, 2012; Shah P. K., Gerasimenko, Roy, N, \& al, 2013). In FNR we still have electrostimulation that can stimulate afferent fibers at the dorsal level of spinal cord, and therefore stimulate the automatic rhythmic movements of CPGs (Musienko, Courtine, \& al, 2012) and reduce spasticity (Hahm, Yoon, \& Kim, 2014). This will make faster the recovery as well as the resistance training with the treadmill incline (Harkema, Schmidt, \& al, 2012; Steinberg, 2013), as well as a gait stimulation over 90 minutes, that will stimulate learning and increase range of motion (ROM) (Kirkby K. A., 2013; Kirkby K. , 2014), 
and even locomotion exercises within the under water treadmill, allowing the first manifestation of voluntary and involuntary movements, due to the properties of water as the floatability, resistance, viscosity and surface tension (Levine, Millis, Flocker, \& MacGuire, 2014; Monk, 2007). To complete we still proved the importance of laser therapy to promote and advance the water locomotor training (Rossmeisl, White, \& al, 2013; Medina, 2013) (Cross, 2014; Kirkby K. , 2014; Newark, 2012).

\section{Conclusion}

With this article it can be concluded that the bipedal LT should be done first and only after patients have the ability to perform the training with $60 \%$ of independence is that the quadrupedal training should be executed. In quadrupedal training, the coordinated movement between the superior and inferior limbs stimulates the connective interneuron. The stimulation caused by the movement of the forelimbs activates the spinal cord-cortex-spinal cord loop, which in turn stimulates motor neuron pathways, increasing PICs that will modulate lumbosacral motor neurons.
In the article it can be concluded that the LT should start with 20 minutes and scaling up to 90 minutes. During the LT on treadmill or underwater treadmill (Figure 4), the speed, the water line and the slope should be equally increased. On the underwater treadmill, water beams with turbulence must be applied to increase sensorymotor inputs as well as any neural circuit.

In the article, it is concluded that the Panjabi's model (Vangelder, Vaughn, \& Hoogenboom, 2013) should be applied as soon as possible, with the greatest intensity, always respecting the passive or ligamentous subsystem.

This article suggests the use of robotic systems such as the Lokomat and its possible application in quadruped animals, especially the dog, since, after 2 to 3 months, with basic LT, the fictive locomotion appears, being sufficient to confer independence to this patients.

Once the similarities regarding the type of hernia, clinical signs, diagnosis and treatment are constant between the dog and human, it is interesting to approach this robotic form of bipedal or quadrupedal LT, and even in the cat, since the neuroplasticity occurs mainly in the rubrospinal tract, but also in the corticospinal tract as in the human.

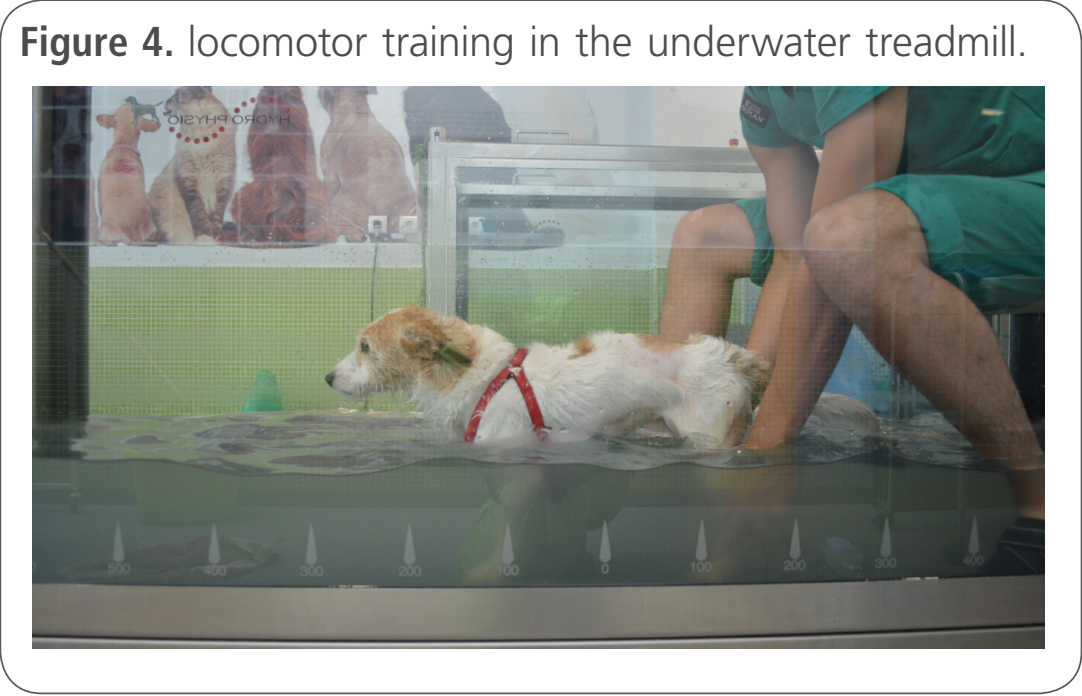




\section{References}

1. Alexeeva, Sames, Jacobs, Hobday, Mitchell, Distasio, \& Calancie. (2011). Comparison of training methods to improve walking in persons with chronic spinal cord injury: a randomized clinical trial. Journal Spinal Cord Medicine.

2. Angeli, Edgerton, R., Gerasimenko, Y., \& Harkema. (2014). Altering spinal cord excitability enables voluntary movements after chronic complete paralysis in humans. Brain.

3. Barros, P., \& Pinheiro, J. (2014). Doenças da Medula Espinhal. Em M. J. Sá, Neurologia Clínica. Universidade Fernando Pessoa.

4. Bostrom, A., et al. (2014). The finish neurological function test battery for dogs and its intra and inter rater reliability. 8th International Symposium on Veterinary Rehabilitation / Physical Therapy and Sports Medicine - American College of Veterinary Sports Medicine and Rehabilitation.

5. Chang, C. W. (2014). Design and Fabrication of a Multi-electrode Array for Spinal Cord Epidural Stimulation. International IEEE.

6. Cross, C. A. (2014). Nose to tail application of red and infrared therapeutic laser for the athletic (and non athletic) dog. International Canine Sports Medicine Symposium.

7. Davies, L. (2015). Rehabilitation of the spinal patient. BSAVA Congress.

8. Dietz. (2012). Neuronal plasticity after a human spinal cord injury positive and negative effects. Experimental Neurology.

9. Ditunno, Cardenas, Dalal, et al. (2012). Advances in rehabilitation management of acute spinal cord injury. Handbook clinic neural Elsevier BV.

10. Dragone, L. (2015). La fisioterapia del paziente spinale Congresso Internazionale - SCIVAC.

11. Duffell, Brown, \& Mirbagheri. (2014). Interventions to reduce spasticity and improve function in people with chronic incomplete spinal cord injury: distinctions revealed by different analytical methods. Neurorehabilitation Neural Repair.

12. Duffell, L. D., \& Mirbagheri, M. M. (2015). Facilitatory effects of anti spastic medication on robotic locomotor training in people with chronic incomplete spinal cord injury. Journal of neuroengineering Rehabilitation.

13. Field-Fote, \& Roach. (2011). Influence of a locomotor training approach on walking speed and distance in people with chronic spinal cord injury: a randomized clinical trial. Phys. Ter.

14. Gad, P., Edgerton, R., et al. (2012). Forelimb EMG - based trigger to cortrol an eletronic spinal bridge to enable hindlimb stepping after a complete spinal cord lesion in rats. Journal Neuroeng Reab.

15. Garcia, G., Alias, Truorg, K., Shah, P. K., Roy, R. R., \& Edguton, V. R. (2015). Plasticity of subcortical pathways promote recovery of skilled hard function in rats after corticospinal and rubrospinal tract injuries. In Brain. Elsevier.

16. Gerasimenko, Y., et al. (2010). Novel and direct access to the human locomotor spinal circuitry. Journal Neuroscience.
17. Hagen, E. (2015). Acute complications of spinal cord injuries. World journal of orthopedics.

18. Hahm, Yoon, \& Kim. (2014). High frequency transcutaneous eletrical nerve stimulation alleviates spasticity after spinal contusion by inhibiting activated microglia in rats. Neurorehabilitation neural repair.

19. Harkema, S. J., \& Behrman, A. L. (2012). Balance and Ambulation Improvements in individuals with chronic-Imcomplete Spinal cord Injury using Locomotor training based rehabilitation. Arch Phys Med Rehab.

20. Harkema, S. J., Schmidt, M., et al. (2012). Establishing the neuro recovery network: multisite rehabilitation centers that promote activity - based therapies and assessements for neurologic disorders. Arch Phys Med Rehab.

21. Harkema, Schmidt-Read, Lorenz, \& Edgerton, R. (2012). Balance and ambulation improvements individuals with chronic incomplete spinal cord injury using locomotor training - based rehabilitation. American Congress of rehabilitation medicine.

22. Hettlich, B., \& Levine, J. M. (2012). Early reherniation of disk material in eleven dogs with surgically treated thoracolumbar intervertebral disk extrusion. Veterinary surgery.

23. JDitunno, Cardenas, Dalal, et al. (2012). Advances in rehabilitation management of acute spinal cord injury. Handbook clinic neural Elsevier BV.

24. Kim, B., Ahn, J., Yoon, B., et al. (2014). Rehabilitation with osteopathic manipulative treatment after lumbar disc surgery: A randomized controlled pilot study. Journal of osteopathic medicine, Elsevier.

25. Kirkby, K. (2013). Rehabilitation of paw injuries. International Canine Sports Medicine Symposium.

26. Kirkby, K. (2014). Lasertherapy. 8th International Symposium on Veterinary Rehabilitation / Physical Therapy and Sports Medicine - American College of Veterinary Sports Medicine and Rehabilitation.

27. Kirkby, K. (2014). Therapeutic exercises . 8th International Symposium on Veterinary Rehabilitation / Physical Therapy and Sports Medicine - American College of Veterinary Sports Medicine and Rehabilitation.

28. Knikou, M. (2012). Plasticity of corticospinal neural control after locomotor training in human spinal cord injury. Neural Plasticity.

29. Lavron, et al. (2015). Activation of spinal locomotor circuits in the decerebrated rat by spinal epidural and/or intraspinal eletrical stimulation. Brain Research.

30. Lavron, I., Gerasimerko, Y., et al. (2014). Activation of spinal locomotor circuits in the decerebrated rat by spinal epidural and/or intraspinal eletrical stimulation. Science Direct, Elsevier.

31. Levine, D., Millis, D., Flocker, J., \& MacGuire, L. (2014). Aquatic therapy. In D. Levine, \& D. Millis, Canine Rehabilitation and Physical therapy. Elsevier.

32. Medina, C. (2013). Clinical applications of class 4 lasertherapy International Canine Sports Medicine Symposium.

33. Millis, D., Garr, \& Weng. (2013). Exercises in canine physical 
rehabilitation: range of motion of the forelimbs during stair and ramp ascent. Journal Small Animals Practice - BSAVA.

34. Monk, M. (2007). Hydrotherapy. In C. McGowan, L. Goff, \& N. Stubbs, Animal Physiotherapy - Assessement, treatment and rehabilitation of animal. Wiley.

35. Musienko, P., Courtine, G. et al. (2012). Somatosensory cortical of balance during locomotion in decerebrated cat. Journal of Neurophysiology.

36. Nas, K., Yazmalar, L., Aydin, A., Ones, K., et al. (2015). Rehabilitation of spinal cord injuries. World journal orthopedic.

37. Newark, B. A. (2012). Latest research and science related to therapeutic lasers. International Canine Sports Medicine Symposium.

38. Nielsen, J., et al. (2015). Leveraging biomedical informaties for assessing plasticity and repair in primate spinal cord injury. Brain Research.

39. Olby, N. (2014). Abordages diagnostico y terapeutico de las patologias neurologicas en pacientes geriatricos caninos. Curso de neurologia en pequenos animales - Novotech.

40. Oosterhuis, T., Ostelo, R., et al (2013). Effectiveness and cost effectiveness of rehabilitation after lumbar disc surgery (realise) design of a randomized controlled trial. BMC Musculoskeletal Disorders - Biomed Central.

41. Rossmeisl, J., White, et al (2013). Acute adverse events associated with ventral slot decompression in 546 dogs with cervical intervertebral disc disease. Veterinary Surgery.

42. Roy, R. R., \& Edgerton, R. (2012). Neurobiological perspective of spasticity as occurs after a spinal cord injury. Experimental Neurology Journal.

43. Roy, R. R., Harkema, J., \& Edgerton, R. (2012). Basic concepts of activity based interventions for improved recovery of motor function after spinal cord injury. Arch Phys Med Rehab.

44. Shah, P., Gerasimenko, Y., Roy, R., \& Tillakaratne. (2013). Use of quadrupedal step training to re engage spinal interneuronal networks and improve locomotor function after spinal cord injury. Brain.

45. Solopova, I. A., et al (2015). Tapping into rhythm generation circuitry in humans during simulated weihtlessness conditions. Frontiers in Systems Neuroscience.

46. Stanley, \& Kim. (2013). Advances in the treatment of cranial cruciate ligament insufficiency in dogs. International Canine Sports Medicine Symposium.

47. Steinberg, H. S. (2013). Neurology of the canine joint. International Canine Sports Medicine Symposium.

48. Takao, T., Tanaka, N., Yanagi, H., et al (2015). Improvement of gait ability with a short term intensive gait rehabilitation program using body weight support treadmill training community dwelling chronic post stroke survivors. Journal of Physical Therapy Science.

49. Tansey. (2010). Neural plasticity and Locomotor recovery after spinal cord injury. PM\&R.

50. Thompson, \& Walpaw. (2014). The simplest motor skill:
Mechanisms and applications of reflex operant conditioning. Exercise Sport Science review.

51. Tillakaratne, N., Edgerton, R., Roy, R. R., et al (2014). Identification of interneurons activated at different inclines during treadmill locomotion in adult rats. Journal of neuroscience research.

52. Vangelder, L., Vaughn, D., \& Hoogenboom. (2013). A phased rehabilitation protocol for athletes with lumbar intervertebral disc herniation. International Journal of sports physical therapy.

53. Visintin, \& Barbeau. (1989). The effets of body weight support on the locomotor pattern os spastic paretic patients. Canadien Journal Neurology Science.

54. Werning, Muller, Nanassy, \& Cagol. (1995). Laufband therapy based on "rules of spinal locomotion" is effective in spinal cord injured persons. European Journal Neuroscience.

55. Wirz, Zemon, Rupp, Scheel, \& Hornby. (2005). Effectiveness of automated locomotor training in patients with chronic incomplete spinal cord injury: a multicenter trial. Arch Phys Med Rehabil.

56. Zhong, H., Roy, R., Leon, R. D., \& Edgerton, R. (2012). Acommodation of the spinal cat to a tripping perturbation. Frontiers in physiology.

\section{Comment on this article:}

\section{8 in $8+8$ P}

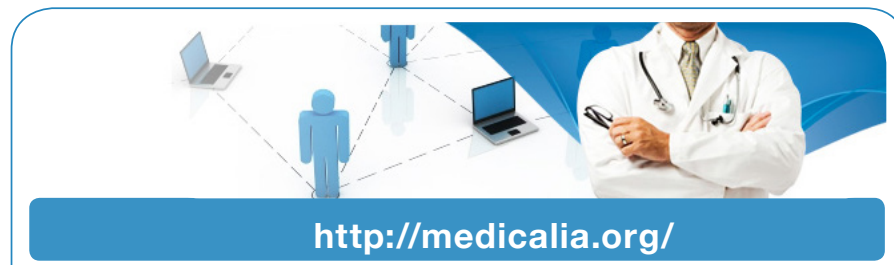

Where Doctors exchange clinical experiences, review their cases and share clinical knowledge. You can also access lots of medical publications for free. Join Now!

\section{Publish with iMedPub}

\section{http://www.imed.pub}

International Archives of Medicine is an open access journal publishing articles encompassing all aspects of medical science and clinical practice. IAM is considered a megajournal with independent sections on all areas of medicine. IAM is a really international journal with authors and board members from all around the world. The journal is widely indexed and classified Q1 in category Medicine. 